\title{
A NONLINEAR MULTIGRID METHOD FOR THE EFFICIENT SOLUTION OF THE STEADY EULER EQUATIONS
}

\author{
P.W.Hemker, B.Koren and S.P.Spekreijse \\ CWI, Centre for Mathematics and Computer Science \\ P.O.Box 4079, 1009 AB Amsterdam, The Netherlands
}

\begin{abstract}
An efficient iterative method has been developed for the accurate solution of the non-isenthalpic steady Euler equations for inviscid flow.

First, the system of conservation laws is space-discretized by a first order finite-volume Osher-discretization. Without time stepping, the steady equations are solved by iteration with nonlinear multiple grid cycles, where a Symmetric Gauss-Seidel method is used as a relaxation. Initial estimates are obtained by the Full Multigrid method. In the pointwise relaxation, the equations corresponding to each cell are kept in block-coupled form, i.e. a Collective Symmetric Gauss-Seidel relaxation is used. In this relaxation local linearization of the equations and the boundary conditions is applied, and one (or a few) step(s) of a Newton iteration is (are) used for the approximate solution of these small nonlinear systems. The first order Osher-discretization has many good properties which foster the efficiency of multigrid iteration. It appears that for all meshsizes the discrete system is solved up to truncation error accuracy in only a few ( 1 to 3 ) iteration cycles ( 3 to 8 work units).

To obtain higher accuracy, we use second order finite volume schemes (e.g. the newly developed superbox scheme [ 3 ]), again based on Osher's approximate Riemann solver. The more accurate discretizations are less stable, and hence harder to solve by relaxation iteration. Therefore, we make use of the fact that the solution of the first order scheme can be computed very efficiently, and we solve the second order system (up to truncation error) by one or a few cycles of a defect correction process.
\end{abstract}

\section{INTRODUCTION}

For a 2-D domain $\Omega^{*}$, we solve the system of non-isenthalpic Euler equations

$$
\begin{aligned}
& \frac{\partial}{\partial t} q+\frac{\partial}{\partial x} f(q)+\frac{\partial}{\partial y} g(q)=0, \\
& q=\left(\begin{array}{c}
\rho \\
\rho u \\
\rho v \\
\rho e
\end{array}\right), \quad f=\left(\begin{array}{c}
\rho u \\
\rho u^{2}+p \\
\rho u v \\
\rho u H
\end{array}\right), \quad g=\left(\begin{array}{c}
\rho v \\
\rho v u \\
\rho v^{2}+p \\
\rho v H
\end{array}\right) ;
\end{aligned}
$$

where $\rho, u, v, e, p$ and $H=e+p / \rho$ represent density, velocity component in $\mathrm{x}$ - and $\mathrm{y}$-direction, specific energy, pressure and specific enthalpy. For a 
perfect gas, (1.1) is completed by

$$
p=(\gamma-1) \rho\left(e-\frac{1}{2}\left(u^{2}+v^{2}\right)\right),
$$

in which $\gamma$ is the ratio of specific heats. In symbolic form we write (1.1) as

$$
q_{t}+N(q)=0 .
$$

The steady equations are obtained by the assumption $q_{t}=0$.

To construct a nested sequence of discretizations for our multigrid solution procedure, we use the finite volume technique. We divide the domain $\Omega^{*}$ in quadrilateral cells $\Omega_{i j}$, such that a mapping is introduced from a regular and rectangular "computational domain" to the irregular "physical domain". By regular refinement of the computational domain, this mapping generates the coordinates for the cell vertices in a sequence of refining irregular grids. To prove the accuracy of the resulting schemes, we take this mapping non-singular and sufficiently smooth.

The discrete approximation $q_{h}$ of $q(x, y)$ is represented by a (vector-) quantity $q_{i j}$ for each $\Omega_{i j}$. Each $q_{i j}$ is associated with the mean value of $q$ over $\Omega_{i j}$. The space discretization now requires the approximation of $\int_{\Gamma}\left(f . n_{x}+g . n_{y}\right) d s, k=N, E, S, W$, at the four walls $\Gamma_{i j k}$ of cell $\Omega_{i j}$. Each wall $\Gamma_{i j k}$ may be either a common boundary with a neighbouring cell $\Omega_{i j k}$ or a part of the boundary $\partial \Omega^{*}$. In both cases the integral is approximated by $f^{k}\left(q_{i j}^{k}, q_{i j k}^{k}\right) \cdot \operatorname{meas}\left(\Gamma_{i j k}\right)$, i.e. at each $\Gamma_{i j k}$ we approximate $f n_{x}+g n_{y}$ by a constant value, only depending on $q_{i j}^{k}$ and $q_{i j k}^{k}$, which are approximations to $q(x, y)$ at $\Gamma_{i j k}$ in $\Omega_{i j}$ and $\Omega_{i j k}$ respectively. First and second order schemes are obtained by different choices for these approximations.

Thus, the discretization of the steady equation (1.2) is the set of nonlinear equations

$$
\left.N_{h}\left(q_{h}\right)\right|_{i, j}:=\sum_{k=N, E, S, W} f^{k}\left(q_{i j}^{k}, q_{i j k}^{k}\right) \operatorname{meas}\left(\Gamma_{i j k}\right)=0 .
$$

for all $(i, j)$ with $\Omega_{i j} \subset \Omega^{*}$.

By the rotation invariance of the Euler equations, we can relate $f^{k}(.,$.$) to a$ local coordinate system, rotated such that it is aligned with $\Gamma_{i j k}$. Then we find $f^{k}\left(q_{i j}^{k}, q_{i j k}^{k}\right)=T_{i j k}^{-1} f\left(T_{i j k} q_{i j}^{k}, T_{i j k} q_{i j k}^{k}\right)$. Here, the operator $T_{i j k}$ takes care of the local rotation of the coordinate system at $\Gamma_{i j k}$ and $f(.,$.$) is a numerical flux$ function, independent of the orientation of $\Gamma_{i j k}$. For $f(.,$.$) we use the numeri-$ cal flux function as proposed by Osher [6]. For details see [4] .

\section{THE FULLY IMPLICIT NONLINEAR MULTIGRID METHOD}

Most methods developed so far for the solution of the steady equations (1.1) are based on integrating the equation (1.2) in time until a steady state is reached. We disregard the time-dependence, and assume that a suitable space discretization takes into account the proper characteristic directions in $\Omega^{*}$, and that for $h \rightarrow 0$ the discrete solution $q_{h}$ approaches an (existing) steady solution $q(x, y)$ that satisfies the entropy condition. Hence, we restrict ourselves to the 
direct solution of the nonlinear system

$$
N_{h}\left(q_{h}\right)=0 \text {. }
$$

For this solution we apply the nonlinear multiple grid (FAS-) algorithm.

We construct the nested set of refining grids, such that each set of $2 \times 2$ cells in a fine mesh forms a single cell in the next coarser mesh.

Slightly generalising the equation (2.1) to

$$
N_{h}\left(q_{h}\right)=r_{h},
$$

where $r_{h}$ denotes a possible correction term, we select a (nonlinear) relaxation procedure

$$
q_{h}^{(n+1)}:=S_{h}\left(q_{h}^{(n)}, r_{h}\right)
$$

for its iterative solution.

The coarser grids are used to accelerate this basic procedure. For this a coarse grid correction is used: starting with an approximation $q_{h}^{(k)}$ on the fine mesh and some approximation $q_{2 h}^{\text {old }}$ on the next coarser, an approximate solution for the coarse grid problem

$$
N_{2 h}\left(q_{2 h}^{\text {new }}\right)=N_{2 h}\left(q_{2 h}^{\text {old }}\right)-\bar{R}_{2 h, h}\left(N_{h}\left(q_{h}^{(k)}-r_{h}\right) ;\right.
$$

is computed. Then the value $q_{h}^{(k)}$ is updated by

$$
q_{h}^{(k+1)}=q_{h}^{(k)}+P_{h, 2 h}\left(q_{2 h}^{\text {new }}-q_{2 h}^{\text {old }}\right) .
$$

The equations (2.4) and (2.5) describe the coarse grid correction step.

Our FAS-cycles for the solution of (2.2) consist of the following steps:

(0) Start with an approximate solution $q_{h}$.

(1) Improve $q_{h}$ by application of a (pre-) relaxation sweep (2.3).

(2) If the present grid is the coarsest, skip to (3); otherwise improve $q_{h}$ by application of one coarse-grid-correction step, where the approximate solution of (2.4) is effected by application of a single FAS-cycle to this coarser grid problem.

(3) Improve $q_{h}$ by another (post-) relaxation sweep (2.3).

For the FAS-procedure, we obtain an initial estimate by the Full Multi-Grid (FMG-) technique [2] : the initial estimate is obtained by interpolation from the approximate solution on the next coarser grid. For many problems this process gives very good results, even if one starts with rough approximations on a really coarse grid [ 5 ].

With a particularly simple restriction $\bar{R}_{2 h, h}$ and prolongation $P_{h, 2 h}$ as transfer operators between the coarse and fine grids, the coarse discrete operator $N_{2 h}$ is a Galerkin approximation to the (first-order) fine grid discretization $N_{h}$. Viz. with $P_{h, 2 h}$ the piecewise constant interpolation over cells, and $\bar{R}_{2 h, h}$ the summation of the residual over $2 \times 2$ fine mesh cells to form a residual on the corresponding coarse cell, we find

$$
N_{2 h}\left(q_{2 h}\right)=\bar{R}_{2 h, h} N_{h}\left(P_{h, 2 h} q_{2 h}\right)
$$


This formula has an interesting implication for a coarse grid correction. Viz. if (2.4)-(2.5) transform the approximation $q_{h}$ into $\tilde{q}_{h}$, the residual of $\tilde{q}_{h}$ generally satisfies

$$
\bar{R}_{2 h, h}\left[r_{h}-N_{h}\left(\tilde{q}_{h}\right)\right]=\mathcal{O}\left(|| q_{h}-\tilde{q}_{h}||^{2}\right) .
$$

This means that after the coarse grid correction step the residual mainly contains high frequency components.

A necessary property of a relaxation method in a multiple grid context is the capability to damp high frequency components in the residual. To ensure this, the discretization should be sufficiently dissipative. For the first order scheme, well-known and simple nonlinear relaxation procedures such as Collective Symmetric Gauss-Seidel work well. ("Collective" means that the 4 variables corresponding to a single cell are relaxed simultaneously.) In most applications we use CSGS in one diagonal direction as pre- and CSGS in the other diagonal direction as post-relaxation. The smoothing behaviour of the relaxations can be analyzed by local mode analysis. If we study plots of reduction factors of Fourier components (spectral radii, or norms for the error or residual amplification operator), we see that two CSGS-sweeps are usually sufficient for a significant reduction of the high frequencies (Hemker, unpublished results). For second order schemes the smoothing rates are not satisfactory.

Wanting at least second order accuracy, we start with a first order approximation $q_{h}^{(1)}$, obtained by a single sweep of the FMG-process, and improve the accuracy by a defect correction process (DCP) $[1,3]$

$$
N_{h}^{1}\left(q_{h}^{(n+1)}\right)=N_{h}^{1}\left(q_{h}^{(n)}\right)-N_{h}^{2}\left(q_{h}^{(n)}\right) .
$$

Here $N_{h}^{p}, \mathrm{p}=1,2$, denotes the $\mathrm{p}$-th order discretization. For smooth solutions a single step of (2.7) is sufficient to obtain the higher order of accuracy [2] . But also, for solutions with discontinuities (where the formal order of convergence has no practical meaning) it is seen that one or a few steps of (2.7) improve the accuracy of the solution significantly, even if the new iterands are approximated by only a few FAS-cycles $[3,5]$. The first iterand $q_{h}^{(2)}$ of $(2.7)$ can also be approximated by application of one additional FMG-sweep that reduces the error by another factor $h$. This means that only two FMG-sweeps may solve the second order equations sufficiently accurate.

3. RESULTS

As standard testcases we consider the NACA0012-airfoil at $M_{\infty}=0.63$, $\alpha=2^{\circ}$ (subsonic flow), and at $M_{\infty}=0.8, \alpha=1.25^{\circ}$ (transonic flow with shock). As a finest grid we use a $128 \times 32$ O-type mesh with an outer boundary at approx. 100 chord lengths away from the airfoil (fig.1). At the outer boundary we impose unperturbed flow. As 2 nd order scheme we use the superbox scheme $[3,5]$. In fig. 2 and 3 we present results and make a comparison with solutions from [7] .

In fig.2a and $3 \mathrm{a}$, the convergence histories of the lift and drag coefficent are shown. As starting point, $q_{h}^{(1)}$, we use a single-FAS FMG-approximation of 
the first order scheme. The lift and drag as published in [7] are spread over the shaded areas. Clearly visible is the excellent improvement of the drag which is obtained in the first DCP-cycle. Taking the results from [7] as a standard, we see that we need 3 DCP-cycles for the subsonic flow, and only 1 DCP-cycle for the transonic flow with a shock.

In fig. $2 b$ and $3 b$, the left graphs show the pressure distributions obtained after the 3rd DCP-cycle, the right graphs are taken from [7]. For the subsonic flow the good agreement is evident. Due to scattering in shock position, this agreement is less for the transonic flow with shock. For the latter the superbox scheme yields solutions of good quality in the smooth parts of the flow, but (being non-TVD) it introduces some spurious non-monotonicity.

For the multigrid computation of airfoil flows with the steady Euler equations, DCP is found to be an efficient solution method for stable 2nd order discretizations. It appears that it is sufficient to perform only a few DCPcycles in which all sub-problems (2.2) are solved by a single FAS-cycle.

\section{ACKNOWLEDGEMENT}

The investigations were supported in part by the Netherlands Technology Foundation.

\section{REFERENCES}

1. K. Böhmer and P. Hemker, and H.J. Stetter (1984). The Defect Correction Approach. Computing Suppl.5, 1-32.

2. W. HaCKBUSCH (1985). Multigrid Methods and Applications, Springer Series Comp. Mathematics, 4, Springer Verlag, Berlin, Heidelberg.

3. P.W. Hemker (1985). Defect correction and higher order schemes for the multigrid solution of the steady Euler equations, CWI Report NM-R8523, To appear in Proceedings Multigrid Conference, Cologne, Oct. 1985.

4. P.W. Hemker and S.P. SPeKreIJSE (1985). Multiple Grid and Osher's Scheme for the Efficient Solution of the Steady Euler Equations, CWI Report NM-R8507, To appear in Appl. Num. Math. 1986.

5. B. KOREN (1986). Evaluation of second order schemes and defect correction for the multigrid computation of airfoil flows with the steady Euler equations, To appear.

6. S. OSHer (1981). Numerical solution of singular perturbation problems and hyperbolic systems of conservation laws. O. Axelsson, L.S. Frank AND A. VAN DER SLUIS (eds.). Analytical and Numerical Approaches to Asymptotic problems in Analysis, Springer Series Comp. Mathematics, North Holland Publ. Comp..

7. A. RuzzI and H. Viviand (1981). Numerical Methods for the computation of inviscid transonic flows with shock waves, Springer Series Comp. Mathematics, Vieweg Verlag, Proceedings GAMM Workshop, Stockholm, 1979. 

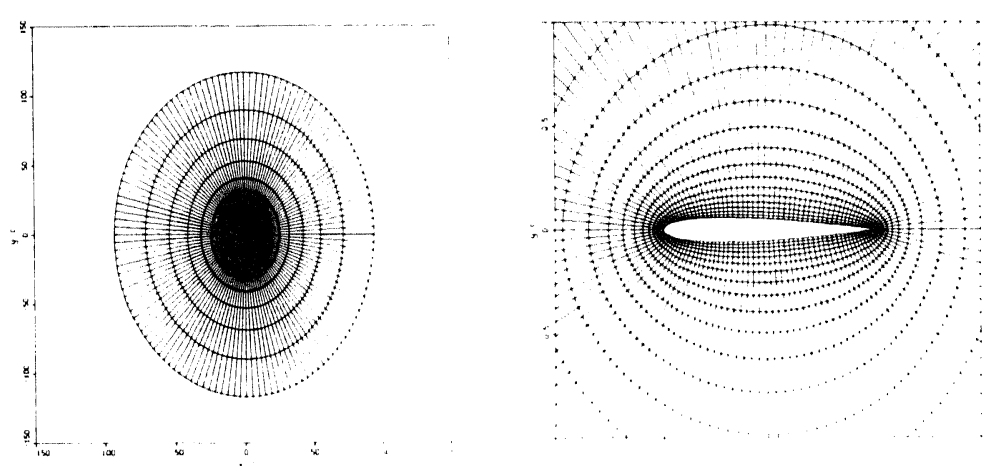

Fig.1: $128 \times 32$-grid NACA0012-airfoil.

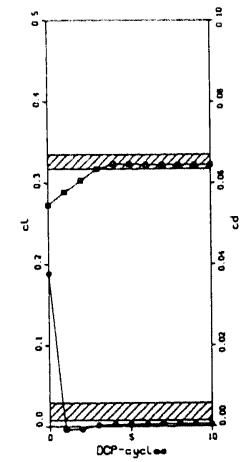

a. Convergence history lift (square) and drag (circular) coefficient

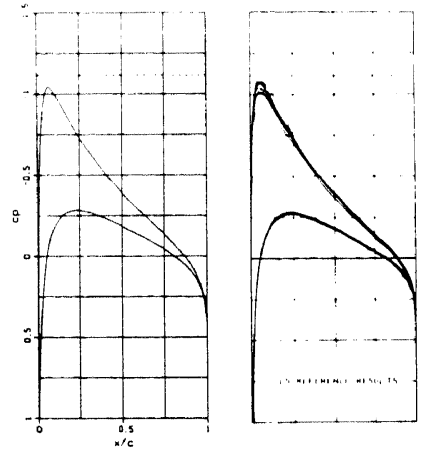

b. Surface pressure distributions

Fig.2: Results for NACA0012-airfoil at $M_{\infty}=0.63$ and $\alpha=2^{\circ}$.

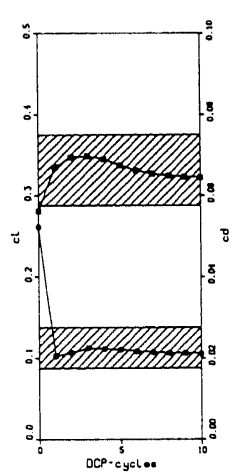

a. Convergence history lift (square) and drag (circular) coefficient
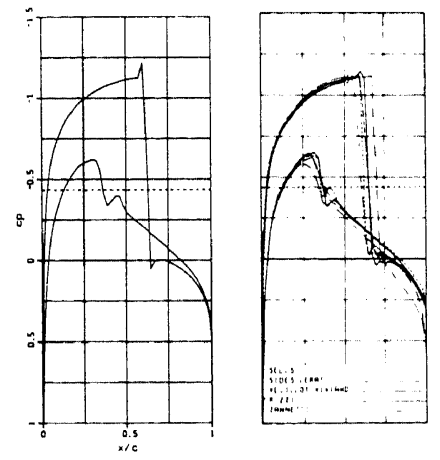

b. Surface pressure distributions

Fig.3: Results for NACA0012-airfoil at $M_{\infty}=0.8$ and $\alpha=1.25^{\circ}$. 